\title{
Parameter Optimization and Experimental Analysis of Passive Energy Storage Power-Assisted Exoskeleton
}

\author{
Ming Han, Baojun Shi $\mathbb{D}^{\text {, }}$, Shijie Wang, Tiejun Li, Jianbin Feng, and Tao Ma \\ School of Mechanical Engineering, Hebei University of Technology, Hebei 300132, China \\ Correspondence should be addressed to Baojun Shi; shibj2003@126.com
}

Received 10 June 2020; Revised 5 October 2020; Accepted 19 October 2020; Published 3 November 2020

Academic Editor: Francesco Aggogeri

Copyright (c) 2020 Ming Han et al. This is an open access article distributed under the Creative Commons Attribution License, which permits unrestricted use, distribution, and reproduction in any medium, provided the original work is properly cited.

\begin{abstract}
To address the occurrence of lumbar spine disease among labor workers who carry heavy objects, a passive energy storage based exoskeletal apparatus was designed to assist, using springs as energy storage elements and utilizing the change in energy that occurs when the human body is bent during the process of lifting objects. First, the mechanism of the exoskeleton was statically modeled; the spring stiffnesses and the locations of support points were used as design variables to optimize the model by optimizing the effective moment on the lumbar spine. Next, an optimized algorithm (Optdes-Sqp) based on the Newton method for solving quadratic programming subproblems was applied to optimize the stiffnesses of compression and extension springs and the positions of the upper support points of each spring. The accuracy of the simulated model was also verified using MATLAB software. Finally, the effect of optimization was verified, and the respiratory rates and heart rates of subjects before and after wearing the exoskeleton were analyzed. The experimental results show that the exoskeleton designed in this study assisted the subjects, and the results lay the foundation for follow-up designs and studies of exoskeletons.
\end{abstract}

\section{Introduction}

Migrant workers engaged in labor-intensive industries such as manufacturing and construction are often required to carry heavy objects, typically weighing $10-20 \mathrm{~kg}$, for extended periods, which results in lumbar spine disease becoming a major health problem for such workers.

In recent years, exoskeleton assistance has been a focus of research in several countries. Lee et al. [1,2] designed a weight-supporting lower extremity exoskeleton (LEE) with a compliant joint and developed a sensor-guided gait-synchronization mechanism, which assists human knee joint movement and reduces knee joint degeneration. Li et al. [3] designed a passive knee-assisting exoskeleton, which stores energy when the knee joint flexes and releases energy when the knee joint extends, to assist motion. Using surface electromyography (sEMG) on the knee joint, their research results show that the exoskeleton could assist the human body when climbing with heavy loads, by decreasing the average maximum load on the knee extensor by about $21 \%$. Panizzolo et al. [4] designed a multi-articular soft exosuit to assist when walking with a heavy load. The exosuit created forces of up to $200 \mathrm{~N}$ at the ankle, for maximum walking speeds of $1.5 \mathrm{~m} / \mathrm{s}(3.4 \mathrm{mph})$, with a total power draw of $59.2 \mathrm{~W}$. Their experimental results show that when the human body wears an exoskeleton for weight-bearing walking, metabolism can be reduced by $6.4 \%$. Zhang et al. [5-7] designed a quasi-passive 3 DOF ankle-foot wearable orthosis and developed a HIT load-carrying exoskeleton (HIT-LEX). Based on human movement identification and control using the HIT-LEX, they proposed a design method for human-machine force interactions. The actual experimentally measured load on the human back was far less than the payload, which showed that the exoskeleton provided good power assistance. Andrikopoulos et al. [8, 9] designed an exoskeletal wrist (EXOWRIST) driven by pneumatic artificial muscles, and its motion was experimentally evaluated via a PID-based control algorithm. Bianchi et al. [10-12] proposed a low-cost hand exoskeleton system (HES) which supports patients suffering from hand opening disabilities during the activities of daily living. Their design process utilized topological optimization (TO) techniques, 
and the test results showed that both opening and closing movements of the hand were facilitated satisfactorily and the natural movements of the fingers were well replicated.

Researchers across the world have also evaluated the assistive effects and performance optimization of exoskeletons. Dijk et al. [13] designed passive energy storage based lower limb exoskeleton, which uses artificial tendons as the energy storage element to assist and optimized the elastic characteristics of tendons to minimize the energy consumption of leg joints during walking. Their simulation results show that the power-assisted exoskeleton reduced the energy consumption by $40 \%$, but, in actual tests, the energy savings were not ideal with the exoskeleton worn by a person walking normally. Zhang et al. [14] optimized device characteristics based on measured human performance. Their research results showed that optimized torque patterns from an exoskeleton worn on one ankle reduced metabolic energy consumption by $24.2 \pm 7.4 \%$ compared to no applied torque. Asbeck et al. $[15,16]$ proposed a multiarticular soft exosuit and a method for evaluating the effect of power assistance. The exosuit had a flexible design to match the joints of the wearer, and the metabolic cost of walking was evaluated by measuring the $\mathrm{O}_{2}$ and $\mathrm{CO}_{2}$ gas exchanges between the subject and the environment. The results showed that accurate force profile tracking was achieved during walking using a real-time admittance controller, and the average physical energy consumption was reduced by $6.4 \%$ when wearing the exosuit with weight-bearing walking. Collins et al. [17] designed a passive ankle exoskeleton, which consisted of an exoskeleton frame, springs, passive clutches, and so forth. When the user of the exoskeleton walks, energy is stored and released using a spring mounted parallel to the lower leg. The optimal spring stiffness coefficient was determined by testing the energy consumption of the human body by experiments conducted with exoskeletons made of springs with different stiffnesses. For the optimal spring stiffness coefficient, the energy consumption while walking was reduced by $7.2 \pm 2.6 \%$ when the exoskeleton was used.

However, the following problems with passive energy storage exoskeletons require further study. (1) In the past, the optimization of the effect of an exoskeleton's assistance only considered the parameter optimization of the energy storage element and did not involve the optimization of the location of the energy storage element. (2) Previous studies rarely evaluated the effect of the exoskeleton in terms of the wearer's respiratory rate and heart rate.

Therefore, this paper designed a passive energy storage exoskeletal apparatus and applied the Optdes-Sqp optimization algorithm to optimize and simulate the stiffness of compression and tension springs, and the positions of their upper supporting points, to fully utilize the body's gravitational potential energy and improve the body energy utilization rate. The correctness of the simulation model was verified using MATLAB software. Finally, from an experimental study on subjects, the changes in respiratory rate and heart rate during the process of lifting heavy objects before and after the exoskeleton was worn were determined, and the optimized enhancing effect of the passive energy storage of the exoskeleton was verified.

\section{Introduction to Exoskeleton Prototype and Construction of Static Model}

2.1. Introduction to Passive Energy Storage Exoskeleton Prototype. As shown in Figure 1, the exoskeleton is designed with a symmetrical structure in the middle, with the upper ends of the breastplate 1 and the backplate 2 connected by a pair of flexible connecting belts 4 , the lower lateral surfaces of the breastplate 1 and the backplate 2 connected by a pair of underarm supports 3, the two compression springs 6 arranged symmetrically and obliquely in a normal splay, the upper hinge support 5 connected and fixed to the breastplate 1 by screws, the lower hinge support 7 connected to the belt 8 by module 9 , and the belt 8 provided with four vertical holes for adjusting the position of module 9 relative to belt 8 .

The two back tension springs 10 are arranged symmetrically and obliquely in an inverted splay, with the upper end and the lower end hook rings of the tension springs 10 , respectively, connected and fixed to the backplate 2 and the belt 8 through a connecting mechanism 11, the backplate 2 provided with four vertical holes for adjusting the position of the upper-end hook rings of the tension springs 10 relative to backplate 2 , and the vertical holes arranged on belt 8 used for adjusting the position of the lower end hook rings of the tension springs 10 relative to belt 8 .

The leg support module 12 is connected to the lower hinge bracket through a pin shaft, and the lower hinge bracket is fixed to module 9 through a screw connection so that the leg support module 12 can rotate relative to the pin shaft. The leg support module 12 is attached to the thigh using a flexible connecting belt to provide support for the upper body mechanism.

The enhancing principle of the passive energy storage exoskeleton is that when a person wears the exoskeleton and bends down to lift a heavy object, the compression spring is gradually compressed, and the back tension spring is gradually extended, thereby converting the reduction in gravitational potential energy into elastic potential energy. When the person lifts the heavy object, the compression and tension springs release the elastic potential energy to compensate for the increase in gravitational potential energy. The trunk gravitational potential energy is thus fully utilized, reducing the energy expended by the person during this process. This, in turn, reduces stress and protects the lumbar vertebra. A schematic diagram of a human body wearing the exoskeleton is shown in Figure 2.

In the past, most exoskeletal systems were active powerassisted mechanisms, which encountered problems such as short battery life and being heavy. Moreover, since these systems mostly use rigid mechanisms, they are uncomfortable for the user. However, the passive energy storage exoskeleton system presented in this paper is made by connecting rigid members using flexible connecting belts, which is comfortable for the user. Also, the mechanism needs no external power and is lightweight, which better 


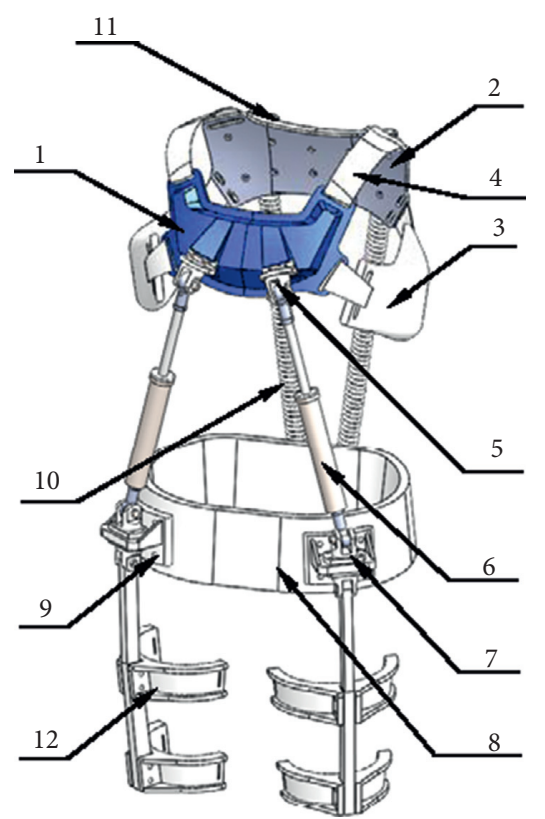

FIgURE 1: Three-dimensional diagram of a passive energy storage exoskeleton mechanism. 1: breastplate; 2: backplate; 3: underarm support; 4: flexible connecting belt; 5 : upper hinge bracket; 6 : compression spring; 7: lower hinge bracket; 8: belt; 9: connection module of the waistband and lower hinge bracket; 10: tension spring; 11: connection mechanism of tension spring shackle, back nail, and waistband; and 12: leg support module.

utilizes the gravitational potential energy of the human torso to aid in lifting heavy objects.

\subsection{Static Modeling of Passive Energy Storage Exoskeleton.} The primary objective of this research is to study the stresses on the human lumbar spine. Hence, a simplified static model of the upper body with the exoskeleton has been developed as depicted in Figure 3.

In Figure $3, \theta_{4}$ and $\theta_{5}$, respectively, represent the included angles between the compression and tension springs and the vertical direction. $F_{1}$ and $F_{2}$, respectively, represent the forces acting on a single compression spring and tension spring and are represented as follows:

$$
\left\{\begin{array}{l}
F_{\mathrm{S} 1}=2 F_{1} \cos \theta_{4}, \\
F_{\mathrm{S} 2}=2 F_{2} \cos \theta_{5}, \\
k_{\mathrm{S} 1}=2 k_{1}, \\
k_{\mathrm{S} 2}=2 k_{2},
\end{array}\right.
$$

where $k_{1}$ and $k_{2}$ represent the stiffness of a single compression spring and tension spring, respectively. Since the process of lifting by a human body is completed in the $x y$ plane, the two tension springs and the two compression springs are equivalent to one tension spring and one compression spring, and $F_{\mathrm{S} 1}, k_{\mathrm{S} 1}$, and $F_{\mathrm{S} 2}, k_{\mathrm{S} 2}$ represent the force and stiffness of the equivalent compression spring and tension spring in the $x y$ plane, respectively.

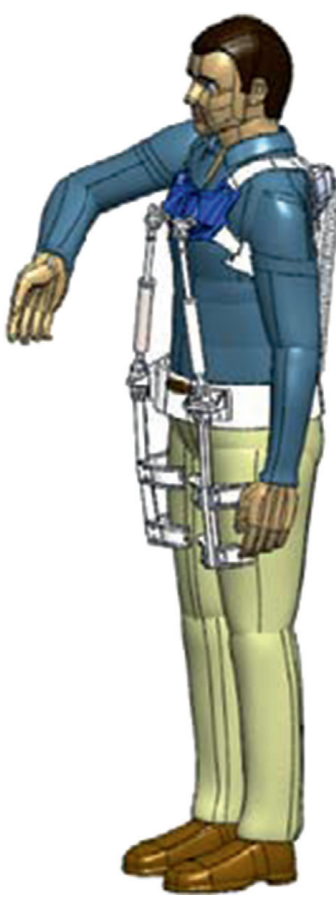

FIgURE 2: Schematic diagram of the exoskeleton on a human body.

Figure 3 is converted into an equivalent $x y$ plane human exoskeleton statics analysis model as shown in Figure 4, where $A_{1} A_{7}$ represents the trunk of a human body of length $l_{17}$ and mass $m_{\mathrm{B}}$. The location of the upper support of the compression spring is $A_{2}$, and the length of $A_{1} A_{2}$ is $l_{12}$. The location of the upper support point of the tension spring is $A_{8}$, and the length of $A_{1} A_{8}$ is $l_{18} . A_{7} A_{9}$ represents the upper arm of the human body with a length of $l_{8}$, and a mass $m_{\mathrm{U}}$. $A_{9} A_{10}$ represents the forearm of the human body of length $l_{9}$ and mass $m_{\mathrm{F}}$. The length of $A_{2} A_{3}$ is $l_{2}$, and the length of $A_{5} A_{8}$ is $l_{5}$. The length of $A_{1} A_{4}$ is $l_{4}$, the length of $A_{1} A_{6}$ is $l_{7}$, and $l_{3}$ and $l_{6}$ represent the lengths of the compression and tension springs, respectively, in real time. $m_{\mathrm{L}}$ represents the mass of the weight lifted by the arms, and $M_{\mathrm{b}}$ is the moment of $A_{1} A_{7}$ rotating shaft.

The spring length relations obtained from Figure 4 are

$$
\left\{\begin{array}{l}
\Delta l_{3}=l_{12}-l_{3} \\
\Delta l_{6}=l_{6}-l_{18}
\end{array}\right.
$$

where $l_{3}$ and $l_{6}$ are the deformations in $l_{3}$ and $l_{6}$, respectively, and $l_{12}$ and $l_{18}$ are the initial lengths of $l_{3}$ and $l_{6}$, respectively. Since the position of the optimal supporting point at the upper end of the two springs is uncertain at this stage, it is assumed that

$$
\left\{\begin{array}{l}
t_{1}=\frac{l_{12}}{l_{17}}, \\
t_{2}=\frac{l_{18}}{l_{17}} .
\end{array}\right.
$$

The statics of the human exoskeleton is represented as follows: 


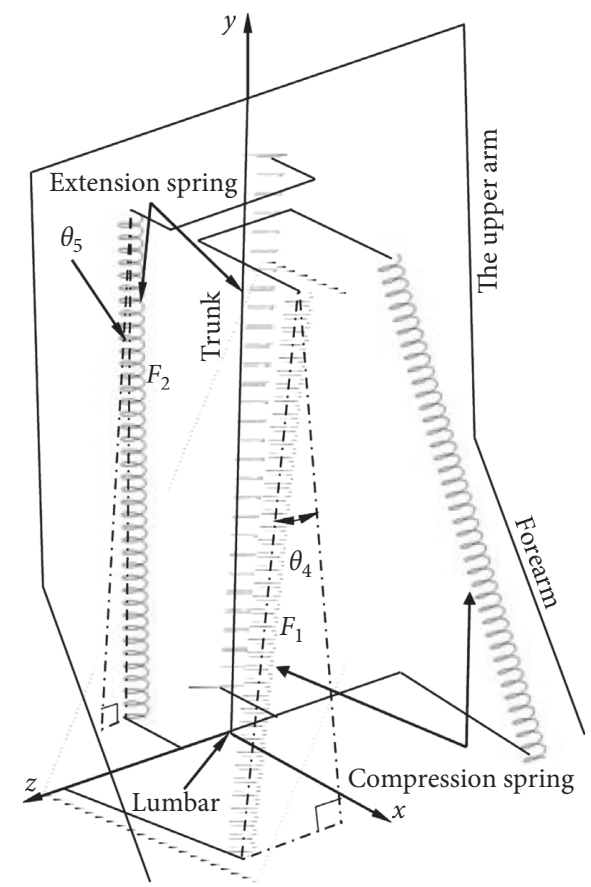

FIgURE 3: Simplified model of the human body with the exoskeleton.

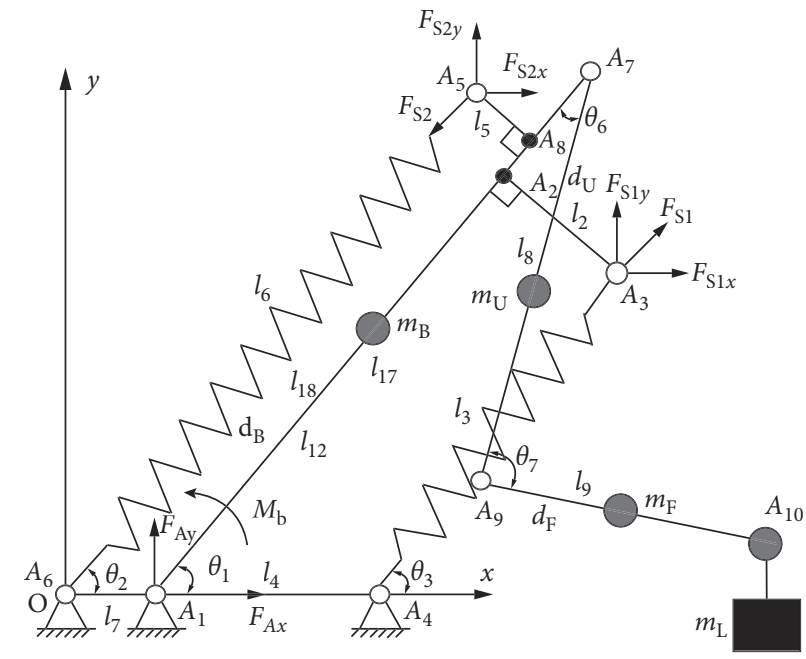

FIGURE 4: Equivalent static model of a human body with the exoskeleton.

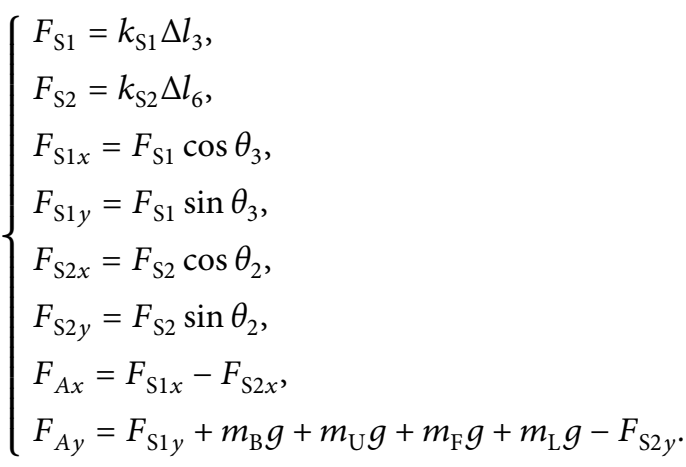

The force components $F_{A x}$ and $F_{A y}$ at point $A_{1}$ along the $x$ and $y$ directions can be obtained by substituting equations (1)-(3) into equation (4):

$$
\left\{\begin{array}{l}
F_{A x}=2\left[k_{1}\left(t_{1} l_{17}-l_{3}\right) \cos \theta_{3}-k_{2}\left(l_{6}-t_{2} l_{17}\right) \cos \theta_{2}\right] \\
F_{A y}=2\left[k_{1}\left(t_{1} l_{17}-l_{3}\right) \sin \theta_{3}-k_{2}\left(l_{6}-t_{2} l_{17}\right) \sin \theta_{2}\right] \\
\quad+m_{\mathrm{B}} g+m_{\mathrm{U}} g+m_{\mathrm{F}} g+m_{\mathrm{L}} g .
\end{array}\right.
$$

From the positional relationship of the centroid of each part, we get 


$$
\left\{\begin{array}{l}
d_{\mathrm{B}}=\left(\frac{l_{17}}{2}\right) \cos \theta_{1}, \\
d_{\mathrm{U}}=l_{17} \cos \theta_{1}-\frac{l_{8}}{2} \cos \left(\theta_{1}+\theta_{6}\right), \\
d_{\mathrm{F}}=l_{17} \cos \theta_{1}-l_{8} \cos \left(\theta_{1}+\theta_{6}\right)+\frac{l_{9}}{2} \cos \left(\theta_{7}-\theta_{1}-\theta_{6}\right), \\
d_{\mathrm{L}}=l_{17} \cos \theta_{1}-l_{8} \cos \left(\theta_{1}+\theta_{6}\right)+l_{9} \cos \left(\theta_{7}-\theta_{1}-\theta_{6}\right) .
\end{array}\right.
$$

In equation (6), $d_{\mathrm{B}}$ represents the horizontal distance between the trunk centroid and point $A_{1}, d_{\mathrm{U}}$ represents the horizontal distance between the upper arm centroid and point $A_{1}, d_{\mathrm{F}}$ represents the horizontal distance between the forearm centroid and point $A_{1}$, and $d_{\mathrm{L}}$ represents the horizontal distance between the weight centroid and point $A_{1}$.

From equation (6), the moment $M_{\mathrm{b}}$ about point $A_{1}$ is obtained as

$$
\begin{aligned}
M_{\mathrm{b}}= & m_{\mathrm{B}} g d_{\mathrm{B}}+m_{\mathrm{U}} g d_{\mathrm{U}}+m_{\mathrm{F}} g d_{\mathrm{F}}+m_{\mathrm{L}} g d_{\mathrm{L}} \\
& -2 F_{2} \cos \theta_{5}-2 F_{1} \cos \theta_{4} .
\end{aligned}
$$

The static model of the passive energy storage exoskeleton worn on the human body is thus established.

\section{Optimal Design of Passive Exoskeleton Parameters}

3.1. Optimization Objectives and Constraints. The purpose of optimization is to determine the optimal combination of four parameters including the stiffnesses $k_{\mathrm{S} 1}$ and $k_{\mathrm{S} 2}$ of the compression and tension springs, respectively, and the position variables $t_{1}$ and $t_{2}$ corresponding to the respective upper-end support points, to minimize the effective moment (which refers to the average of the sum of the absolute values of the moments on the human body when lifting objects), that is, the minimum energy that the human body consumes when lifting heavy objects. The optimization objective function is defined as follows:

$$
\min \tau=f\left(k_{\mathrm{S} 1}, k_{\mathrm{S} 2}, t_{1}, t_{2}\right)=\sum_{i=1}^{N}\left(\left|\frac{M_{\mathrm{b}}(i)}{N}\right|\right),
$$

where $\tau$ represents the effective moment on the human lumbar spine, $f\left(k_{\mathrm{S} 1}, k_{\mathrm{S} 2}, t_{1}, t_{2}\right)$ represents the relationship between the effective moment on the lumbar spine during a lifting task cycle and the four parameters defined above. $N$ represents the number of equal divisions of the task period $T$, and $M_{\mathrm{b}}(i)$ corresponds to the moment $M_{\mathrm{b}}$ acting on the lumbar spine in equation (7) at the $i^{\text {th }}$ division of the task period.
Figure 5 shows the schematic diagrams of the compression and tension springs, where $d$ represents the spring wire diameter and $D$ represents the spring outer diameter. The equation for spring stiffness is represented as follows:

$$
k=\frac{G d^{4}}{8 D^{3} n}
$$

where $k$ is the spring stiffness, $G$ is the shear modulus of the spring material, and $n$ is the effective number of turns of the spring. Based on the above exoskeleton design, the ranges of the outer diameter and spring length of the tension and compression springs are determined. The approximate range of spring stiffness is determined by the corresponding relationship between the effective number of turns of the spring and the wire diameter of the spring. In this study, springs with different stiffnesses were purchased and tested. The results show that if the spring stiffness is too large, the human exoskeleton is difficult to stretch and compress.

In summary, the boundary conditions of the four parameters corresponding to the respective stiffnesses $k_{\mathrm{S} 1}$ and $k_{\mathrm{S} 2}$ of the compression and tension springs, and the position variables $t_{1}$ and $t_{2}$ of the respective upper support points are determined as follows:

$$
\text { s.t. }\left\{\begin{array}{l}
0<k_{\mathrm{S} 1} \leq 3500 \mathrm{~N} / \mathrm{m} \\
0<k_{\mathrm{S} 2} \leq 5000 \mathrm{~N} / \mathrm{m} \\
0 \leq t_{1} \leq 1 \\
0 \leq t_{2} \leq 1 .
\end{array}\right.
$$

3.2. Optimization Methods and Results. This paper focuses on the assistance offered by the exoskeleton to the lumbar spine when the human body lifts $10 \sim 20 \mathrm{~kg}$ of heavy objects. Therefore, a human body lifting a mass of $15 \mathrm{~kg}$ while wearing the exoskeleton is simulated, and the Adams software is used to analyze the moment required to straighten the lumbar spine while lifting the weight, for different stiffnesses of the two springs, and for different positions of their upper support points, from which the optimal combination of parameters for the model are obtained.

The Adams dynamic simulation model established based on Figure 1 is shown in Figure 6.

The length, centroid position, and corresponding mass parameters of the human trunk, upper arm, and forearm are initially set, as shown in Table 1 .

According to experimental measurements and calculations, the total time for a subject to complete a lifting task cycle is about $7 \mathrm{~s}$ and includes $1.25 \mathrm{~s}$ from the beginning of the bending motion to touching the heavy object, $2.25 \mathrm{~s}$ from lifting the heavy object to being fully erect, $2.25 \mathrm{~s}$ from being erect to lowering the heavy object, and $1.25 \mathrm{~s}$ from the bent position to the initial posture. The maximum bending angle of the subject and the angular changes of the elbows and shoulders can also be obtained from this analysis. Therefore, the lumbar motion (Motion 1), shoulder motion (Motion 2), and elbow motion (Motion 3) at the joint of the forearm and 


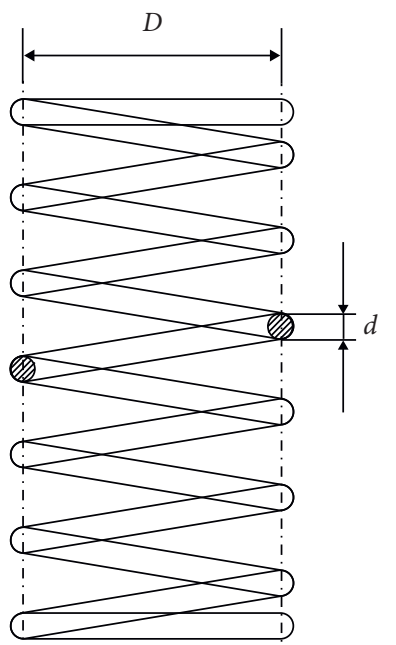

(a)

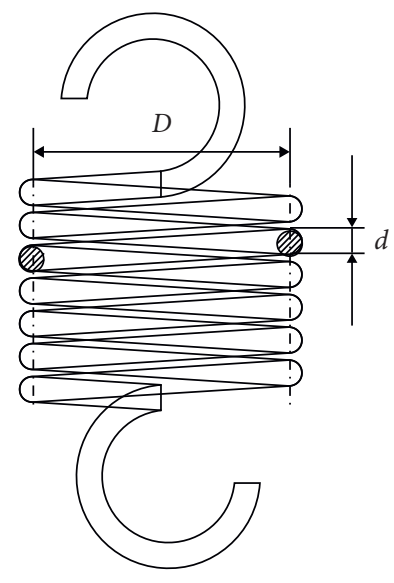

(b)

FIGURE 5: Schematic diagrams of springs used in the exoskeleton. (a) Compression spring. (b) Tension spring.

upper arm are similar to the abovementioned time and positional variations.

The Optdes-Sqp algorithm uses the Newton method to establish a Hessian matrix to solve quadratic programming subproblems. The iterative step size is obtained by solving quadratic programming subproblems, and the optimal solution is obtained iteratively.

In this section, the subject's weight of $67 \mathrm{~kg}$ and a height of $1.67 \mathrm{~m}$ are considered as the weight and height parameters, respectively. The Optdes-Sqp algorithm of the Adams software is used to optimize the parameters. The optimized simulation results are listed in Table 2.

Table 2 shows that the optimized target value is smaller than the present value. The optimized preset values are $k_{\mathrm{S} 1}=0, k_{\mathrm{S} 2}=0, t_{1}=0.5, t_{2}=0.5$, that is, when the human body is not wearing the exoskeleton, the target function (i.e., the effective moment on the lumbar spine) is $99.05 \mathrm{Nm}$, and the target function after the optimization analysis is $38.291 \mathrm{Nm}$, indicating a decrease of $61.34 \%$. The optimal parameters obtained are $k_{\mathrm{S} 1}=2581 \mathrm{~N} / \mathrm{m}, k_{\mathrm{S} 2}=3795 \mathrm{~N} / \mathrm{m}, t_{1}=0.82$, and $t_{2}=0.87$.

\section{Simulation and Experimental Verification of Optimization}

4.1. Validation of Simulation Model. According to equations (5) and (6) of the static modeling of the human exoskeleton, a function file for equation (7) is compiled using MATLAB. According to the angle changes and the time taken by the lumbar spine, elbow, and shoulder of the abovementioned subject, the function file is run to obtain the moment of the lumbar spine when the human body is not wearing the exoskeleton and lifting a $10 \mathrm{~kg}$ weight in a task cycle and is compared with the simulation result obtained from the Adams software. The result is shown in Figure 7.

As can be seen from Figure 7, the change in the moment on the lumbar spine during a lifting task cycle as simulated by MATLAB is almost identical to that obtained from the Adams dynamics software. The maximum value of the moment obtained from MATLAB simulation is $152.925 \mathrm{Nm}$, and the value of the effective moment is $86.69 \mathrm{Nm}$, while the maximum value of the moment obtained by the Adams dynamics simulation is $136.15 \mathrm{Nm}$, and the value of the effective moment is $88.39 \mathrm{Nm}$. Thus, the values of the effective moment are similar. These results verify the accuracy of the simulation model established in this paper.

4.2. Dynamic Simulation Verification of Optimization. The dynamic simulation model of the human body wearing the exoskeleton as shown in Figure 6 is established with the values of the optimized parameters obtained above. The resultant force and moment on the lumbar spine are simulated and solved for the condition that the human body lifts a $15 \mathrm{~kg}$ weight before and after wearing the exoskeleton to complete one experimental lifting task cycle as defined previously. The simulation results are shown in Figures 8 and 9.

The solid and dotted lines in Figures 8 and 9, respectively, represent the resultant force and moment on the lumbar spine during the lifting task cycle, when the human body lifts a $15 \mathrm{~kg}$ weight before and after wearing the exoskeleton. As can be seen from Figure 8, the resultant force on the lumbar spine is significantly reduced. The average value of the resultant force on the lumbar spine before wearing the exoskeleton is $548.31 \mathrm{~N}$, and the average value of the resultant force on the lumbar spine after wearing the exoskeleton is $399.2 \mathrm{~N}$, representing a decrease of $27.2 \%$. As can be seen from Figure 9, the simulation shows that the effective moment on the lumbar spine when lifting a $15 \mathrm{~kg}$ weight without wearing the exoskeleton is $109.68 \mathrm{Nm}$, and the moment on the lumbar spine after wearing the exoskeleton is significantly reduced, with the effective moment being $46.53 \mathrm{Nm}$, representing a decrease of $57.6 \%$, indicating 


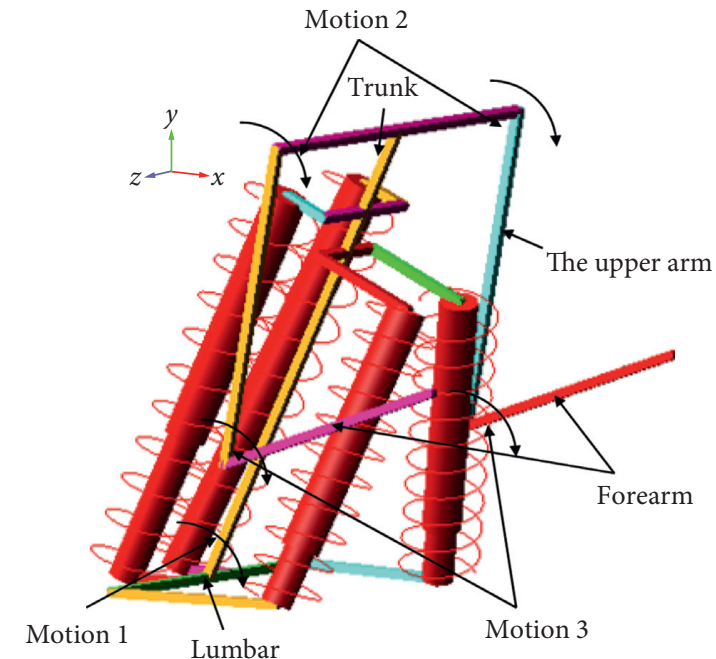

Figure 6: Dynamic simulation optimization model.

TABLE 1: Human model parameters.

\begin{tabular}{|c|c|c|c|}
\hline Part of the human body & Length $(\mathrm{cm})$ & Centroid $(\mathrm{cm})$ & Proportion of body weight (\%) \\
\hline Trunk & $l_{17}=0.3 \mathrm{H}$ & $d_{\mathrm{B}}=0.660 l_{17}$ & 55.7 \\
\hline Upper arm & $l_{8}=0.159 \mathrm{H}$ & $d_{\mathrm{U}}=0.436 l_{8}$ & 2.7 \\
\hline Forearm & $l_{9}=0.146 \mathrm{H}$ & $d_{\mathrm{F}}=0.430 l_{9}$ & 1.5 \\
\hline
\end{tabular}

TABLE 2: Optimization of simulation analysis.

\begin{tabular}{|c|c|c|c|c|c|}
\hline Number of iterations & Measured value $(\mathrm{Nm})$ & $k_{\mathrm{S} 1}(\mathrm{~N} / \mathrm{m})$ & $k_{\mathrm{S} 2}(\mathrm{~N} / \mathrm{m})$ & $t_{1}$ & $t_{2}$ \\
\hline 0 & 99.05 & 0.0000 & 0.0000 & 0.5000 & 0.5000 \\
\hline 1 & 92.114 & 1141.4 & 957.43 & 0.4934 & 0.5000 \\
\hline 2 & 83.538 & 1911.6 & 2564.1 & 0.5002 & 0.5624 \\
\hline 3 & 83.261 & 1974.4 & 2695.0 & 0.5131 & 0.6777 \\
\hline 4 & 73.685 & 2186.8 & 2894.8 & 0.5339 & 0.8537 \\
\hline 5 & 70.130 & 2233.9 & 2985.8 & 0.5799 & 0.8598 \\
\hline 6 & 66.948 & 2315.5 & 3165.7 & 0.6101 & 0.8622 \\
\hline 7 & 56.340 & 2355.6 & 3258.3 & 0.6574 & 0.8653 \\
\hline 8 & 48.645 & 2402.4 & 3355.0 & 0.6831 & 0.8674 \\
\hline 9 & 45.774 & 2437.5 & 3485.4 & 0.7042 & 0.8691 \\
\hline 10 & 39.848 & 2478.5 & 3531.3 & 0.7328 & 0.8694 \\
\hline 11 & 39.433 & 2519.5 & 3621.4 & 0.7524 & 0.8694 \\
\hline 12 & 38.694 & 2551.6 & 3727.7 & 0.8243 & 0.8694 \\
\hline 13 & 38.291 & 2581.1 & 3795.2 & 0.8245 & 0.8694 \\
\hline 14 & 38.291 & 2581.1 & 3795.2 & 0.8245 & 0.8694 \\
\hline
\end{tabular}

that the energy consumption of the lumbar spine is also effectively reduced. Therefore, the exoskeleton developed with the optimized values of the parameters selected for this study has good performance and a significant effect when the parameters are optimized.

\subsection{Experimental Evaluation of the Effect of a Human Body} Wearing an Exoskeleton Prototype. Since it is difficult to directly measure the actual force/moment on the lumbar spine during human motion and since the respiratory rate and heart rate of a subject can indirectly represent the extent of fatigue of the human body during weight lifting, this paper considers the changes in the respiratory rate and heart rate of the subject before and after wearing the exoskeleton to complete the weight lifting task, as the evaluation index of the effect of the aid. We recruited several subjects to lift a mass of $10 \mathrm{~kg}$ and conducted three lifting task experiments on each subject. A COSMED K $4 \mathrm{~b}^{2}$ Exercise Cardiopulmonary Tester was used to collect the respiratory rate and heart rate for each subject before and after wearing the exoskeleton. Each experiment included 20 identical exercise cycles. The experimental setup is shown in Figure 10. 


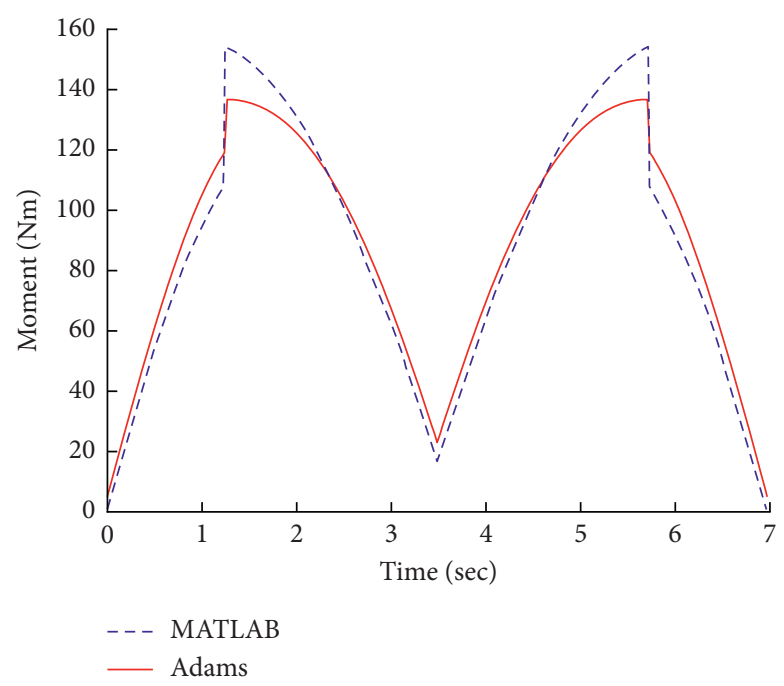

Figure 7: Comparison between the results of MATLAB and Adams software in simulating the moment on a human lumbar spine during a lifting task cycle.

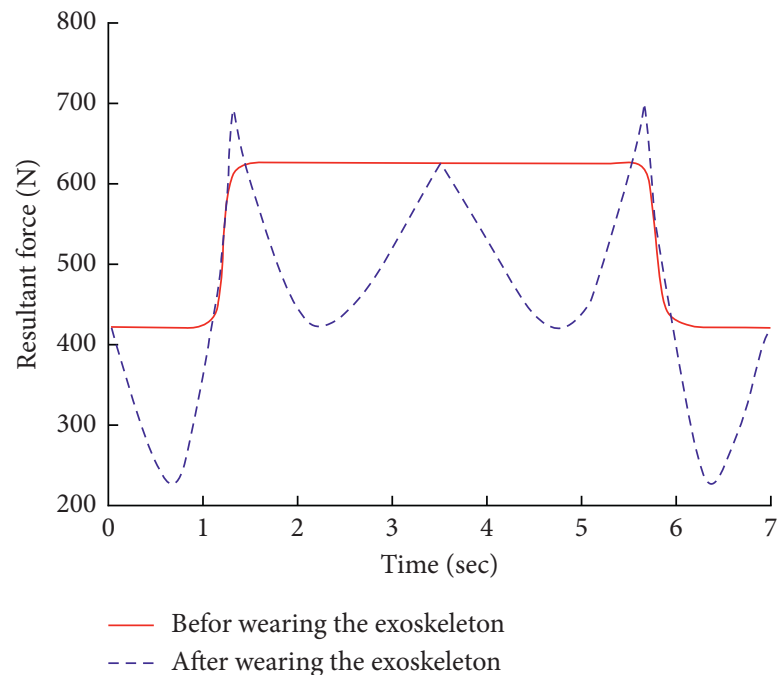

FIGURE 8: Comparison of simulation results of forces on the lumbar spine before and after wearing the exoskeleton.

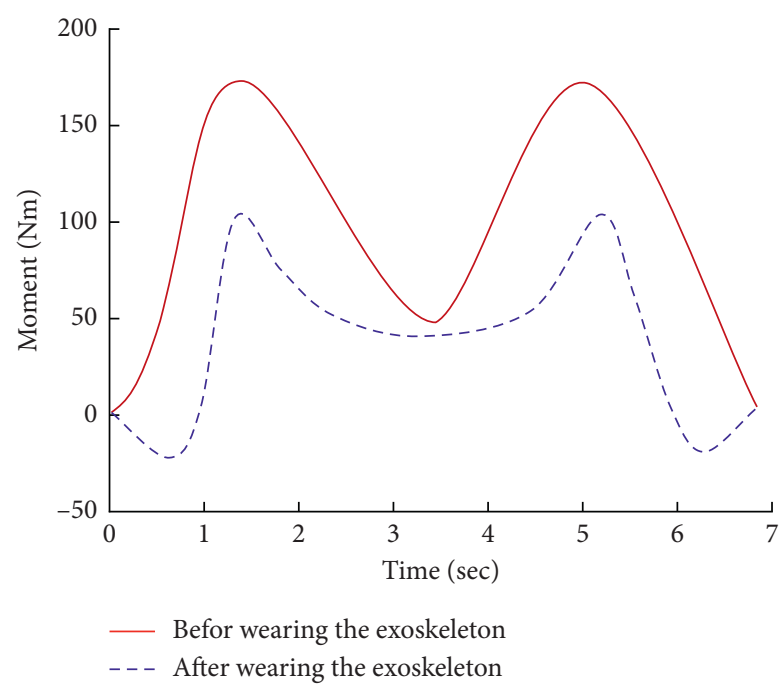

FIGURE 9: Comparison of simulation results of the moment on the lumbar spine before and after wearing the exoskeleton. 


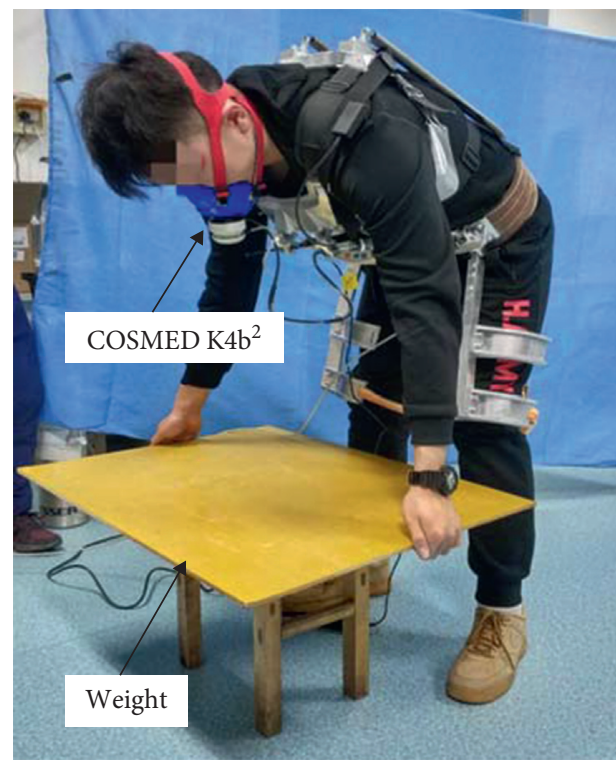

Figure 10: Respiration and heartbeat frequency acquisition experiment.

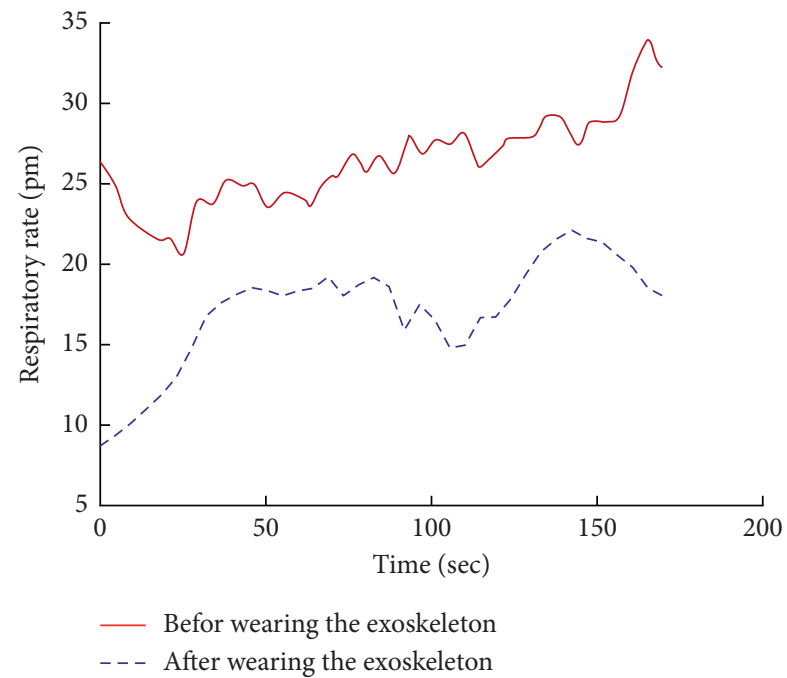

Figure 11: Comparison of respiratory rate of subjects before and after wearing the exoskeleton (third experimental cycle).

Figures 11 and 12 show the changes in the respiratory rate and heart rate of the subjects before and after wearing the exoskeleton during a single experiment, respectively. The red solid line and the blue dashed line in the figures represent the changes in the respiratory rate and heart rate of the subjects before and after wearing the exoskeleton during 20 lifting task cycles, respectively. It can be seen from the figures that the respiratory rate and heart rate decreased after wearing the exoskeleton.

To improve the reliability of the experiment, we recruited three subjects to perform the same tasks of the experiment, and the results are listed in Table 3.

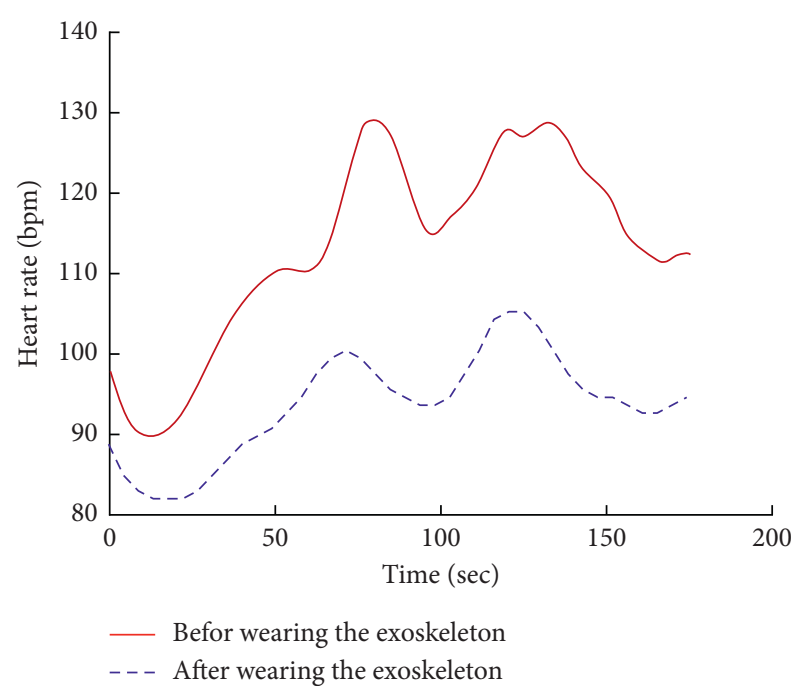

Figure 12: Comparison of the heart rate of subjects before and after wearing the exoskeleton (third experimental cycle).

TABLE 3: Experimental results.

\begin{tabular}{lcc}
\hline $\begin{array}{l}\text { The } \\
\text { subjects }\end{array}$ & $\begin{array}{c}\text { Respiratory rate (pm) } \\
\text { before (after) wearing } \\
\text { exoskeleton }\end{array}$ & $\begin{array}{c}\text { Heart rate (bpm) before } \\
\text { (after) wearing } \\
\text { exoskeleton }\end{array}$ \\
\hline \multirow{2}{*}{$27(15)$} & $117(95)$ \\
& $24(17)$ & $118(90)$ \\
$25(16)$ & $121(102)$ \\
2 & $24(17)$ & $124(104)$ \\
& $26(16)$ & $123(108)$ \\
3 & $25(18)$ & $114(89)$ \\
\hline \multirow{2}{*}{ Average } & $25(14)$ & $101(89)$ \\
\hline
\end{tabular}

\section{Conclusions}

To address the occurrence of lumbar spine disease among labor workers, a passive energy storage exoskeletal apparatus was designed in this study to protect the lumbar spine, and the stiffness and positional parameters of the springs used were optimized to maximize the utilization of human energy during the process of lifting weights. The study results are as follows:

(1) The new passive energy storage exoskeleton designed in this study solves the problems of short life spans and large weights associated with traditional active exoskeletons. The optimization method of energy storage elements and the strategy for evaluating the enhancing effects of the exoskeleton based on the characteristics of the wearer's breathing and heart rate provide a novel method for exoskeleton design and research. 
(2) The built-in Optdes-Sqp algorithm of the Adams software was applied to specifically optimize the parameters of the passive energy storage exoskeleton and simulate the stiffness of the compression and extension springs, as well as the positions of the upper support points of each spring. The optimal parameters obtained were $k_{\mathrm{S} 1}=2581 \mathrm{~N} / \mathrm{m}$, $k_{\mathrm{S} 2}=3795 \mathrm{~N} / \mathrm{m}, t_{1}=0.82$, and $t_{2}=0.87$.

(3) MATLAB software was used to simulate the changes in the moment on the lumbar spine during the lifting of a $10 \mathrm{~kg}$ weight without the exoskeleton, and the results were compared with the simulation results obtained using the Adams. The simulation results showed that the changes in the torque on the lumbar spine obtained by the two methods were almost identical, and the values of the effective moment had little difference. The results thus verify the accuracy of the simulation model established in this paper.

(4) The assistance of the exoskeleton was verified based on the values of the optimized parameters, and the results showed that the resultant force and effective moment on the lumbar spine decreased by $27.2 \%$ and $57.3 \%$, respectively, and the optimization effect was significant. Three subjects were recruited to lift a $10 \mathrm{~kg}$ weight for the experiment. The results showed that the average breathing frequencies of the subjects before and after wearing the exoskeleton were $25 \mathrm{pm}$ and $16 \mathrm{pm}$, and average heart rates were $114 \mathrm{bpm}$ and $94 \mathrm{bpm}$, respectively. After wearing the exoskeleton, the respiratory rate and heart rate of the subjects decreased by $36 \%$ and $18 \%$, respectively, which proved that the exoskeleton provided assistance and protection to the wearer.

\section{Data Availability}

The data used to support the findings of the study are confidential and are not publicly available.

\section{Conflicts of Interest}

The authors declare that they have no potential conflicts of interest.

\section{Acknowledgments}

This work was supported by the National Key R\&D Program of China (2018YFB1306900), the National Natural Science Foundation of China (U1813222), and the Natural Science Foundation of Hebei Province (E2019202338).

\section{References}

[1] K. M. Lee and D. Wang, "Design analysis of a passive weightsupport lower-extremity-exoskeleton with compliant kneejoint," in Proceedings of the 2015 IEEE International Conference on Robotics and Automation (ICRA), pp. 5572-5577, Seattle, WA, USA, May 2015.

[2] D. Wang and K. M. Lee, "Sensor-guided gait synchronization for weight-support lower-extremity-exoskeleton," in
Proceedings of the 2016 IEEE International Conference on Advanced Intelligent Mechatronics (AIM), pp. 71-76, Banff, Canada, July 2016.

[3] B. Li, B. Yuan, S. Tang et al., "Biomechanical design analysis and experiments evaluation of a passive knee-assisting exoskeleton for weight-climbing," Industrial Robot: An International Journal, vol. 45, no. 4, pp. 436-445, 2018.

[4] F. A. Panizzolo, I. Galiana, A. T. Asbeck et al., "A biologicallyinspired multi-joint soft exosuit that can reduce the energy cost of loaded walking," Journal of Neuroengineering \& Rehabilitation, vol. 13, no. 1, pp. 1-13, 2016.

[5] C. Zhang, X. Zang, Z. Leng et al., "Human-machine force interaction design and control for the HIT load-carrying exoskeleton," Advances in Mechanical Engineering, vol. 8, no. 4, pp. 1-14, 2016.

[6] C. Zhang, Y. Zhu, J. Fan, J. Zhao, and H. Yu, "Design of a quasi-passive 3 DOFs ankle-foot wearable rehabilitation orthosis," Bio-Medical Materials and Engineering, vol. 26, no. 1, pp. S647-S654, 2015.

[7] C. Zhang, G. Liu, C. Li et al., "Development of a lower limb rehabilitation exoskeleton based on real-time gait detection and gait tracking," Advances in Mechanical Engineering, vol. 8, no. 1, pp. 1-9, 2016.

[8] G. Andrikopoulos, G. Nikolakopoulos, and S. Manesis, "Design and development of an exoskeletal wrist prototype via pneumatic artificial muscles," Meccanica, vol. 50, no. 11, pp. 2709-2730, 2015.

[9] G. Andrikopoulos, G. Nikolakopoulos, and S. Manesis, "Motion control of a novel robotic wrist exoskeleton via pneumatic muscle actuators," in Proceedings of the 2015 IEEE 20th Conference on Emerging Technologies and Factory Automation (ETFA), pp. 1-8, Luxembourg City, Luxembourg, September 2015.

[10] M. Bianchi, F. Fanelli, E. Meli, A. Ridolfi, and B. Allotta, "Optimization-based scaling procedure for the design of fully portable hand exoskeletons," Meccanica, vol. 53, no. 6, pp. 3157-3175, 2018.

[11] M. Bianchi, F. Buonamici, R. Furferi, and N. Vanni, "Design and optimization of a flexion/extension mechanism for a hand exoskeleton system," in Proceedings of the ASME 2016 International Design Engineering Technical Conferences and Computers and Information in Engineering Conference, pp. 1-7, Charlotte, NC, USA, December 2016.

[12] M. Bianchi, F. Fanelli, L. Giordani, A. Ridolfi, and B. Allotta, "An automatic scaling procedure for a wearable and portable hand exoskeleton," in Proceedings of the 2016 IEEE 2nd International Forum on Research and Technologies for Society and Industry Leveraging a better tomorrow (RTSI), pp. 287291, Bologna, Italy, September 2016.

[13] W. V. Dijk, H. V. D. Kooij, and E. Hekman, "A passive exoskeleton with artificial tendons: design and experimental evaluation," in Proceedings of the 2011 IEEE International Conference on Rehabilitation Robotics, pp. 1-6, Zurich, Switzerland, June 2011.

[14] J. Zhang, P. Fiers, K. A. Witte et al., "Human-in-the-loop optimization of exoskeleton assistance during walking," Science, vol. 356, no. 6344, pp. 1280-1284, 2017.

[15] A. T. Asbeck, S. M. M. De Rossi, K. G. Holt, and C. J. Walsh, "A biologically inspired soft exosuit for walking assistance," The International Journal of Robotics Research, vol. 34, no. 6, pp. 744-762, 2015.

[16] Y. Ding, I. Galiana, A. Asbeck, B. Quinlivan, and C. Walsh, "Multi-joint actuation platform for lower extremity soft exosuits," in Proceedings of the 2014 IEEE International 
Conference on Robotics and Automation (ICRA), pp. 13271334, Hongkong, China, June 2014.

[17] S. H. Collins, M. B. Wiggin, and G. S. Sawicki, "Reducing the energy cost of human walking using an unpowered exoskeleton," Nature, vol. 522, no. 7555, pp. 212-215, 2015. 